\title{
Pediatric Rehabilitation Medicine in the USA
}

\section{Edward A. Hurvitz, MD, Dennis J. Matthews, MD}

Physiatrists have been taking care of children with disabilities since the beginning of the field. Yet, pediatric rehabilitation medicine is still young; its official recognition commenced in 2003 with the initiation of a certificate of special qualifications. Pediatric physiatrists have enjoyed increasing recognition during the past few years, including greater visibility within the American Academy of Physical Medicine and Rehabilitation (referred to herein as the Academy) with the establishment of the Pediatric Rehabilitation and Developmental Disabilities Council, as well as nationally with greater involvement in organizations such as the American Academy of Cerebral Palsy. A number of challenges remain to the growth of pediatric rehabilitation. Dr. Amy Houtrow, in her report on the survey results of the Council [1], identifies some of these and provides important information that will be useful for planning, as well as a metric to determine growth and improvement in the field. These problems include limited geographic dispersion, slow academic progress, and the lack of federally funded investigators. She also raises interesting questions about gender inequity in compensation.

Children with disabilities are spread throughout the nation, and pediatric rehabilitation medicine specialists provide important care and services for these children. Yet, the survey results revealed a significant concentration of such physicians in the Midwest compared with other regions of the country. The most likely reason for this is the strong pediatric rehabilitation training programs found in the Midwest in cities such as Chicago, Ann Arbor, Detroit, Minnesota, Cincinnati, Kansas City, and others, combined with the strong tendency of trainees to end up practicing not far from where they trained. Furthermore, in wellestablished centers, this value and contribution is highly recognized, creating a greater demand and a need for a larger physician group. However, in many areas of the country, pediatric physiatrists often need to demonstrate the value that they bring to a team caring for pediatric onset disability. The Academy, and especially the Pediatric Rehabilitation and Developmental Disabilities Council, can help by promoting knowledge about the field to our pediatric colleagues.

Dr. Houtrow notes that pediatric rehabilitation medicine physicians may be less academically competitive or academically inclined. The reported academic ranks were: $7 \%$ instructor, $40.7 \%$ assistant professor, $27.9 \%$ associate professor, and $8.1 \%$ professor. An additional $16.3 \%$ reported no academic rank. Of most interest were the facts that $30 \%$ of the assistant professors had been practicing for more than 10 years, and $18 \%$ of the associate professors had been practicing more than 20 years. The possible explanations for this academic stagnation are inadequate mentoring and guidance for promotion, and less individual physician interest in pursuing an academic career. We hypothesize that both are probable factors.

Most (71\%) of the respondents to Dr. Houtrow's survey were women. Previous investigators $[2,3]$ have noted that women in academia face many significant challenges and issues, including balancing work with family, time management, adequate mentors or role models, and gaining credibility from peers and administrators.

This latter issue suggests that the departmental chairs should foster and facilitate academic advancement for those female faculty members inclined to advance their careers. The department leaders should develop family-friendly policies and practices, including flexible work hours, tenure stops, shared positions, modified duties, and clinical activity. They must work to minimize the gender biases in promotion and tenure and especially to increase the reward for clinical service and teaching, assist junior faculty to identify gaps and offer suggestions for strengthening their portfolio, and encourage the faculty to attend their
E.A.H. University of Michigan, Ann Arbor, Ml Disclosure: nothing to disclose

D.J.M. Department of PM\&R, University of Colorado School of Medicine and The Children's Hospital, 13123 East 16th Ave, Aurora, CO 80045. Address correspondence to D.J.M.; e-mail: matthews.dennis@tchden.org Disclosure: nothing to disclose 
university's workshops on tenure and promotion. At present, pediatric rehabilitation medicine has very little funded research and therefore very few mentors.

The field of physical medicine and rehabilitation is attempting to solidify its research productivity. Frontera [4] recommended the development of research capacity by increasing the following: (1) researchers; (2) research culture, environment, and infrastructure; (3) funding; and (4) partnerships. To accomplish this task, issues such as training, mentoring, and placing new investigators must be addressed, as do other issues concerning the recruitment and retention of established investigators.

Far too few programs exist that provide optimal training in medical rehabilitation research or the research infrastructure required to produce successful research. The Rehabilitation Medicine Scientist Training Program of the Association of Academic Physiatrists provides research training, mentorship, and career development support for those physiatrists committed to developing productive careers in academic medicine and research. The ultimate aim of the Rehabilitation Medicine Scientist Training Program is to increase the number of rigorously trained, extramurally competitive, and scientifically productive faculty members in physical medicine and rehabilitation departments. In addition, we recommend partnerships with scientists in other disciplines, academic departments, and institutions, and with consumers with disabilities, among others, that are vital to enhancing the capacity for conducting high-quality, meaningful research.

The gender issue raised in the survey needs further study. The survey asked respondents to adjust part-time salaries to full time, but there is no way to verify whether this step was performed. Although we do not have data, our observation is that many more women choose part-time appointments because of childcare and other needs. Part-time faculty and medical staff often have less opportunity to participate in bonus programs because of limitations on clinic hours and on available time for other pursuits such as higher-level administrative positions and academic work. However, Dr. Houtrow did note that men earn more than women at almost every academic rank and even with the title of medical director. It behooves every chair and practice leader to examine this issue and to determine root causes. In addition to being patently unfair, a true gender discrepancy will not help recruitment in a field that appears to be more attractive to women than to men.

Pediatric rehabilitation, as well as other areas of physiatry that address major disability, also faces the significant challenge of attracting trainees. Fellowships in pain, spine, and sports medicine are blossoming, and an increasing percentage of residents are attracted to PM\&R to pursue a musculoskeletal practice. Medical students interested in caring for children with disabilities will get more exposure to pediatric neurology, and even developmental medicine, through their general pediatric rotations. We must seek ways to increase visibility to students. A strong research base, for example, attracts medical students interested in academics. We could all work on being better mentors, researchers, and ambassadors to students and residents, although it is admittedly difficult when one has a relative value unit target to reach and grant deadlines to meet. Nevertheless, this must become a priority.

To grow as a field, those in the discipline of pediatric rehabilitation medicine must address the problems identified in the survey. We must grow our geographic base so that, across the nation, calling a physiatrist for cerebral palsy or childhood trauma is an expected part of the care plan. We must grow our research base with increased quantity and quality of research, which requires the mentoring to pursue funding and to develop identifiable areas of investigation. We must pursue academic excellence and leadership so that we can develop those mentors. We must certainly address any pay inequities and strive to bring compensation to a competitive level. This survey will require further definition, carefully considered action, and follow-up to measure progress to advance the field.

\section{REFERENCES}

1. Houtrow A. Results From the 2009 Pediatric Rehabilitation Practice Survey of the AAPM\&R Pediatric Rehabilitation/Developmental Disabilities Council. PM R 2010;3.

2. Harrison RA, Gregg JL. A time for change: An exploration of attitudes toward part-time work in academia among women internists and their division chiefs. Acad Med 2009;84:80-86.

3. Mayer AP, Files JA, Ko MG, Blair JE. Academic advancement of women in medicine: Do socialized gender differences have a role in mentoring? Mayo Clin Proc 2008;83:204-207.

4. Frontera WR. Research priorities in rehabilitation medicine. Int $J$ Rehabil Res 2009;32:279. 